\title{
Validity of Improved MTL for Predicting Effective Length of Counterpoise Wire under High-Valued Lightning Currents
}

\author{
Saeed Reza Ostadzadeh \\ Assistant Professor from Engineering department, Arak University, Arak, Iran. \\ *corresponding author, E-mail: $\underline{\text { s-ostadzadeh@araku.ac.ir }}$
}

\begin{abstract}
In this paper, an efficient modeling approach called improved MTL is used to predict effective length of counterpoise wires considering both ionization and dispersion of soils. This paper consists of two parts. At first part, validity of the model for computing effective length of counterpoise wires considering only soil ionization is investigated. The simulation results show that the improved MTL-based effective length of counterpoise wire are in good agreement with the existing formulae. Application of this modeling approach to include ionization and dispersion effects simultaneously (both-affected soil) is carried out in the second part. The simulation results show that in bothaffected soils, the effective length with respect to onlyionized soils, is decreased especially in highly resistive soils under slow-fronted currents. This makes inclusion of both effects financially important in designing counterpoise wires.
\end{abstract}

Keywords: Ionization, dispersion, multi-conductor transmission line, counterpoise wires, lightning strike, and effective length.

\section{Introduction}

Counterpoise wires as typical grounding systems are widely used in discharging lightning currents to the soil. Proper design of such devices are strictly dependent upon including complex phenomena such as ionization of soil [1], frequency variations of electrical parameters of soil (dispersion) [2] and so on. The former is occurred when the electric field of soil surrounding counterpoise wires is exceeded from its critical value inside soil, whereas the latter is taken place when the electrical parameters of soil is frequency-dependent.

There are a number of approaches for analysis and design of grounding systems including the frequency-domain approaches [3-5] for only-dispersive soils, and the timedomain approaches [6-8] for only-ionized soils. In the soils where ionization and dispersion of soils are simultaneously occurred, the mixed frequency-time domain approaches [915] should be used. All mentioned methods above are complex and time-consuming.

One of important parameters in designing counterpoise wires is effective length which is defined as the length beyond which the impulse impedance (the ratio of peak values of induced voltage to lightning current) is no longer varied. In this regard a number of predicting formulae have been proposed for effective length of grounding electrodes in onlyionized soils [16, 17], only-dispersive soils [18, 19], and neither-affected soils $[19,20]$.

In contrast with the mentioned accurate methods, J. L. Guardado et al [22] proposed multi-conductor transmission line model (MTL) and it was validated in soils having constant electrical parameters. In this model, each set of parallel conductors in the grounding systems is assumed as a multi-conductor transmission lines (MTL). A two-port network for each set of parallel conductors in the grounding system is then defined. Finally, the two-port networks are interconnected depending upon the pattern of connections in the grounding system and its representative equations are reduced. Through this approach, voltages and currents at any junction in the grounding system is easily extracted. Since the MTL is in the frequency domain, it was successfully applied in dispersive soil [23]. More recently it has been improved to include nonlinear phenomenon of ionization as well (improved MTL) [24] and was validated by comparing with full-wave methods and experiment. The validity of the proposed model for predicting effective length of counterpoise wires, however, was not investigated. J. L. He et al [16] based on numerical solution of transmission line method in time domain proposed closed-form solutions for effective length of counterpoise wires buried in only-ionized soils. As well known, combining dispersion effect with the predicting formulae in [16] is very difficult. Hence, it is still a challenge to be investigated.

In this study, validity of the improved MTL for predicting effective length of counterpoise wires considering only ionization of soil is first investigated. The simulation results proves good agreement and very short run-time in comparison with [16]. In addition, since the proposed approach is in the frequency domain, the soil dispersion effect can be easily included. Impact of both effects with respect to situation where only ionization effect is occurred, results in decreasing effective length that should be financially considered in designing such grounding systems especially in highly resistive soils.

This paper is organized as follows. In section II, principles of the modified MTL is briefly explained with emphasis on the counterpoise wires. Section III is focused on validity of the modified MTL for predicting effective length of 
counterpoise wires buried in only-ionized soils. In section IV, significance of both effects on the effective length of counterpoise wires is investigated. Finally, concluding remarks are given in section $\mathrm{V}$.

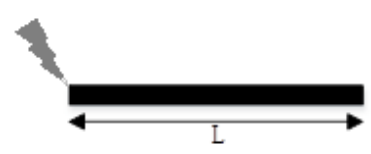

(a)

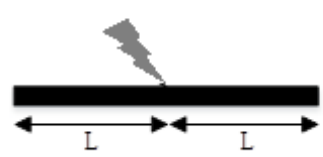

(b)

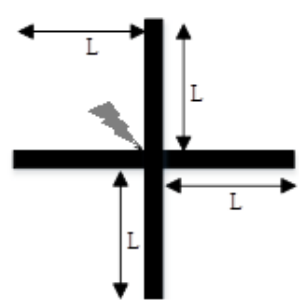

(c)

Figure 1: Different arrangements of horizontal electrodes under lightning current. (a): corner-injected one-arm electrode, (b):center-injected two-arm electrode, (c): centerinjected four-arm electrode.

\section{Improved MTL}

Consider a horizontal electrode under high-valued impulse current so that ionization phenomenon is taken place as shown in figure 2(a). This phenomenon is conventionally modelled as gradually increasing radius as shown in figure 2(b) in which the electrode is divided into $\mathrm{N}$ segment of length $1_{k}$ and equivalent radius $a_{k}[8]$. Then, the sending and receiving voltage and current for each segment is defined as shown in figure 3(a). After then, the relation between voltage and current at the sending and receiving points is represented as the two-port network as shown in figure 3(b) and expressed in (4).

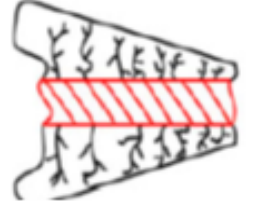

(a)

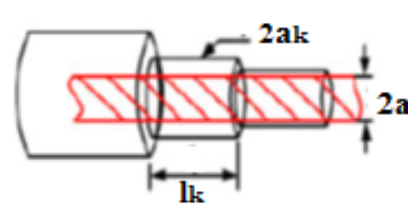

(b)
Figure 2: (a): Ionization phenomenon around the conductor, and (b): ionization model as gradually increasing radius. Adapted from [8].

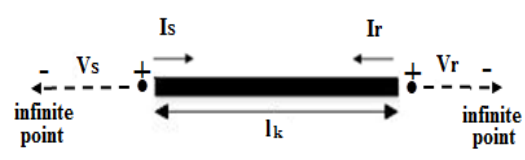

(a)

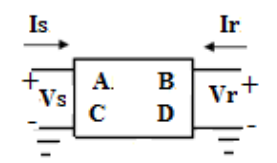

(b)
Figure 3: (a): Definition of sending and receiving voltage and current for a conductor of length $1_{k}$ and (b): Two-port network representation of a conductor of length $l_{k}$.

$$
\left[\begin{array}{c}
I_{s k} \\
I_{r k}
\end{array}\right]=\left[\begin{array}{cc}
A_{k} & B_{k} \\
C_{k} & D_{k}
\end{array}\right]\left[\begin{array}{c}
V_{s k} \\
V_{r k}
\end{array}\right]
$$

where $V_{\text {sk }}$ and $I_{\text {sk }}$ represent, respectively, the voltage and current at the sending point of the kth segment, and $V_{\text {rk }}$ and $\mathrm{I}_{\mathrm{rk}}$ are, respectively, the voltage and current at the receiving point of the $k$ th segment at each frequency inside spectral content of lightning current.

Note that the defined matrix is different with transmission matrix in microwave multi-port network theory. Now, if the above representation is applied to each segment in figure 2(b), and the lightning current is also denoted by current source ( $I_{s}$ ), the cascaded two-port networks as shown in figure 4 is consequently achieved.

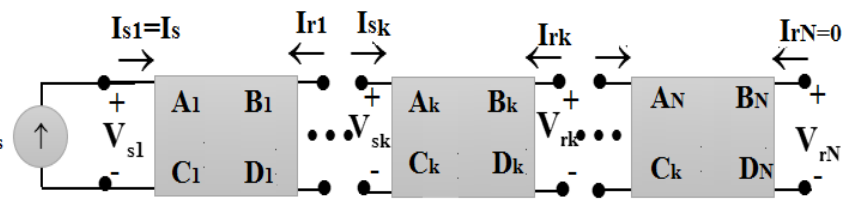

Figure 4: Cascade connection of two-port networks for figure 2(b).

After a few mathematical manipulations, the voltage at the sending and receiving points of all segments are related to each other as expressed in (5) [24].

$\overline{\mathrm{V}}_{\mathrm{s}}=\overline{\overline{\mathrm{MTL}}}^{-1} \overline{\mathrm{I}}_{\mathrm{s}}$

where $\overline{\mathrm{I}}_{\mathrm{s}}$, and $\overline{\mathrm{V}}_{\mathrm{s}}$ are vectors including sending and receiving currents and voltages of all segments. Also, matrix of $\overline{\overline{\text { MTL }}}$ includes two-port networks of all segments. From (5), once the lightning current $\left(\mathrm{I}_{\mathrm{s}}\right)$ is known, the sending and receiving voltages at any segment can be easily computed. Also, the sending and receiving currents of each segment are computed from the individual two-port network as expressed in (4).

The perpendicular component of electric field on the surface of kth segment is then computed as bellow [24]

$E_{k}=\frac{I_{L k} / 1_{k}}{2 \pi(1 / \rho+j 2 \pi f \varepsilon) a_{k}}, \quad k=1,2, . ., N$

Where $\varepsilon$ and $\rho$ are dielectric constant and resistivity of the lossy soil. $\mathrm{I}_{\mathrm{Lk}}$ is also leakage current of $k t h$ segment computed via subtracting currents at the sending and receiving points of the each segment. If the value of $E_{k}$ is greater than its critical value $\left(\mathrm{E}_{\mathrm{c}}\right)$ [15], radius of each segment is increased as

$\mathrm{a}_{\text {new }, \mathrm{k}}=\mathrm{a} \times \mathrm{E}_{\mathrm{k}} / \mathrm{E}_{\mathrm{c}}, \quad \mathrm{k}=1,2 \ldots, \mathrm{N}$

For the new value of radius, Eq. (5) is again solved up to $\mathrm{E}_{\mathrm{k}}<\mathrm{E}_{\mathrm{c}}$ for each frequency inside spectral content of the lightning current. Finally, the sending voltage of each 
conductor in time domain, $\mathrm{v}_{\mathrm{sk}}(\mathrm{t})$ is computed as follows

$\mathrm{v}_{\mathrm{sk}}(\mathrm{t})=\sum_{\mathrm{m}=1}^{\mathrm{M}} \mathrm{V}_{\mathrm{sk}, \mathrm{m}} \cos \left(2 \pi \mathrm{f}_{\mathrm{m}} \mathrm{t}+\phi_{\mathrm{m}}\right)$

Where $\mathrm{M}$ denotes the total number of frequencies from spectral content of lightning current. Further information in more detail for improved MTL can be found in [24].

As explained in [22], the mutual coupling effect among parallel conductors is only considered, and the other ones, i.e. echelon and collinear couplings, are low enough so that they can be neglected. Therefore, the analysis of center-fed electrodes with two and four arms are the same as corner-fed one-arm electrode except that the lightning current is equally divided into arms.

\section{Validity}

In this section, the validity of the proposed method for analyzing grounding electrodes buried in only-ionized soils is investigated. Hence, a center-fed four-arm electrode (cross-arm electrode) with length of $\mathrm{L}=5 \mathrm{~m}$ which is injected by an impulse current $20 / 40 \mu$ s with peak value of $7 \mathrm{kA}$, is considered. The soil is a horizontal two-layer media in which the upper and lower layer resistivity are respectively $15.8 \Omega \mathrm{m}$ and $2.6 \Omega \mathrm{m}$. The upper layer thickness is also 6.2 $\mathrm{m}$. The simulation results for transient voltage based on the improved MTL is shown in figure 5. In this figure, the measured results [11] are also included (see figure 5 in [11]). From this figure, one can easily observe that although the mutual coupling among collinear arms is ignored, good agreement is achieved. To better comparison, the peak value of transient voltage, i.e. grounding potential rise (GPR), and rise time of the transient voltages are compared in table 1 . From this table, good agreement is observed.

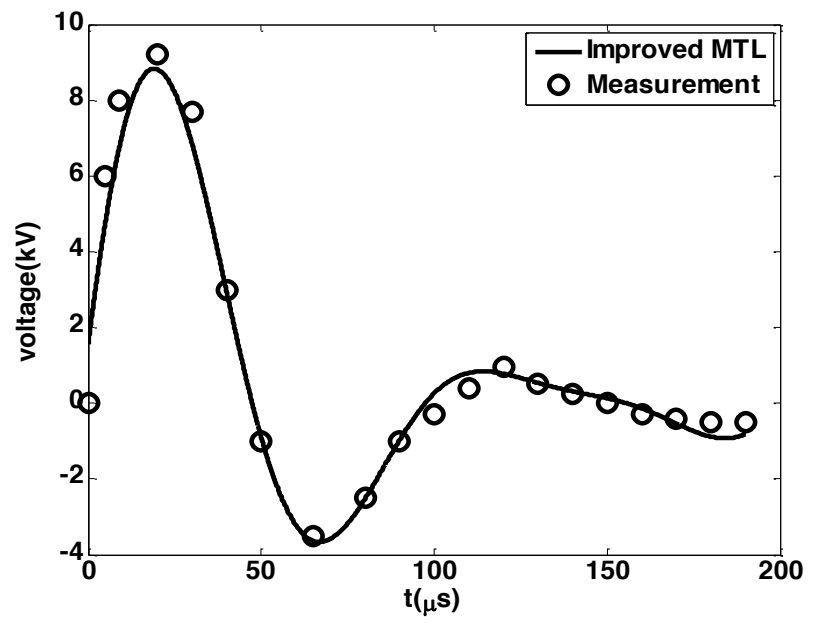

Figure 5: Transient voltage of center-fed four-arm electrode based on the improved MTL and measurement.

Table 1: GPR and $T_{r}$ of center-fed four-arm electrode based on improved MTL and measurement.

\begin{tabular}{c|cc}
\hline Cross-arm electrode & $\mathrm{GPR}(\mathrm{kV})$ & $\mathrm{T}_{\mathrm{r}}(\mu \mathrm{s})$ \\
\hline Improved MTL & 9.2 & 19.5 \\
\hline Measurement [11] & 8.8 & 20 \\
\hline
\end{tabular}

\section{MTL-Based Analysis of Counterpoise Wires}

In this section, the improved MTL is applied to counterpoise wires in figure 1 , and its validity on the effective length in only-ionized soils is investigated. To this end, a horizontal electrode of length $12 \mathrm{~m}$ and radius $12.5 \mathrm{~mm}$ buried in a lossy soil with different resistivity and relative dielectric constants [25] is selected. The lightning current is also the same as [25] which is shown in figure 6 . The transient voltage of counterpoise wires with and without considering ionization for different lossy soils are shown in figure 7. As seen, the simulation results of corner-fed one-arm electrode in figure 7(a) is in excellent agreement with [25]. Also, from figures 7(b) and (c), when the number of arms are increased the ionization effect is decreased especially for poorly resistive soils. This is owing to decreasing the current injecting into the arms which results in decreasing the ionization effect. The impulse impedances (the ratio of peak values of transient voltage to injected current) are computed as shown in figure 8 . The effective length, i.e. a starting length at which the impulse impedance is no longer varied [26], is easily extracted as arrows in figure 8 and compared with the individual ones in [16] as shown in figure 9.

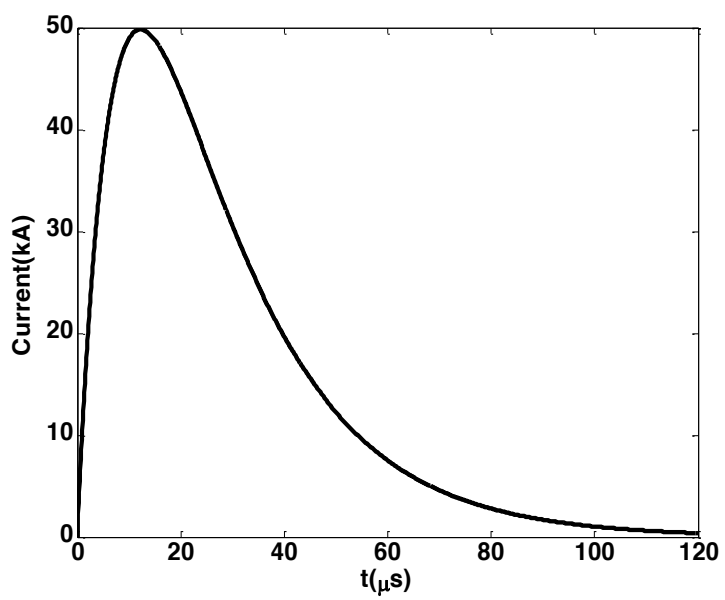

Figure 6: Time domain representation of the lightning current used in this study.

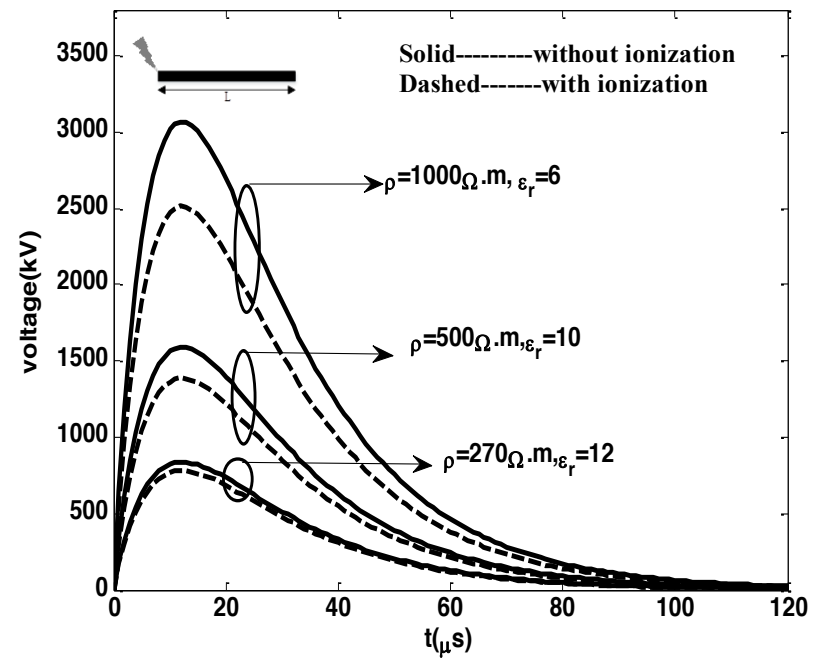

(a) 


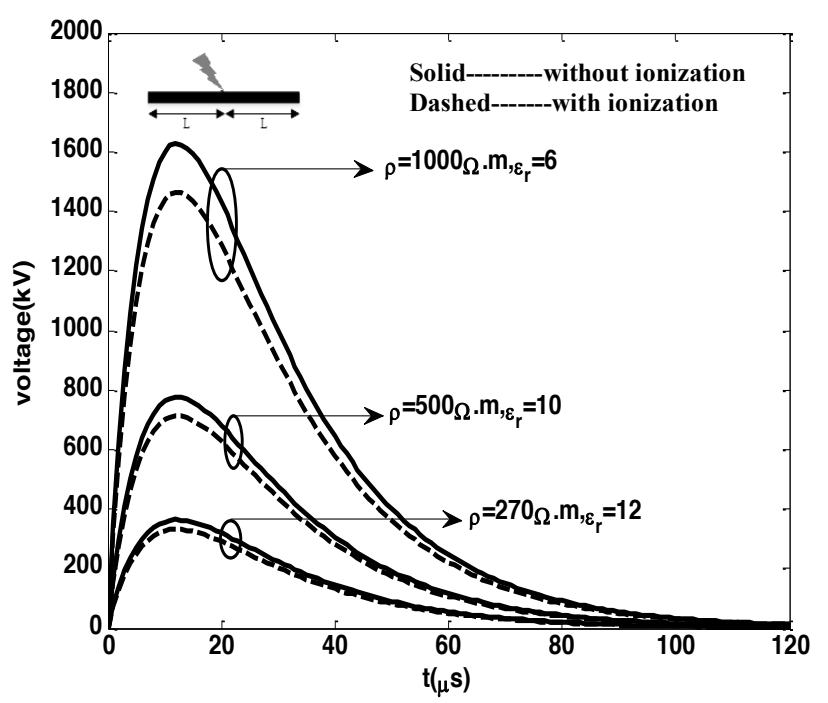

(b)

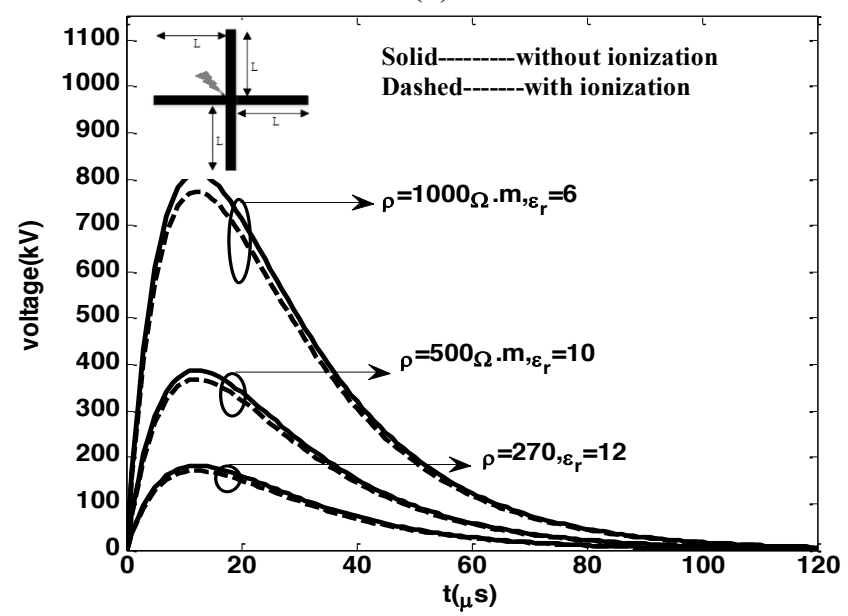

(c)

Figure 7: Transient voltage of (a): corner-fed electrode, (b): center-fed electrode with two arms, and (c): center-fed electrode with four arms for the three lossy soils with and without considering ionization of soil.

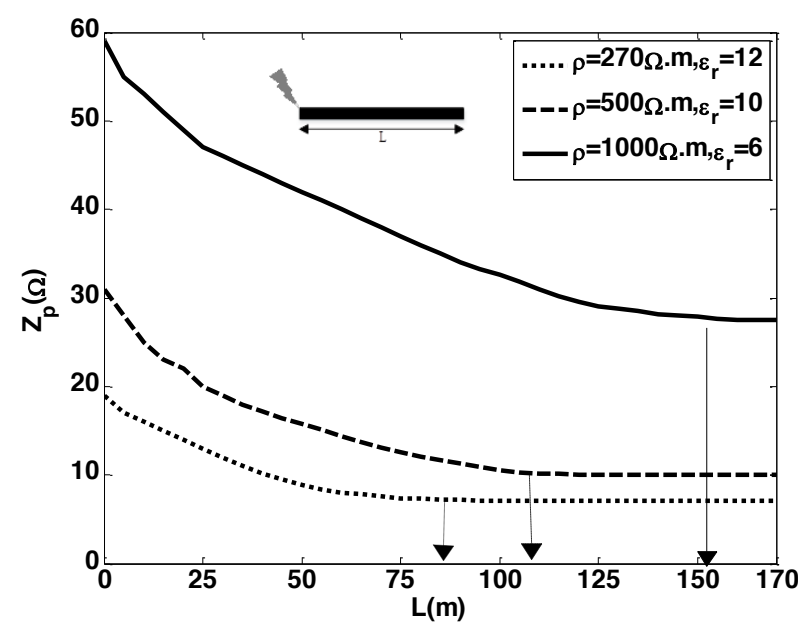

(a)

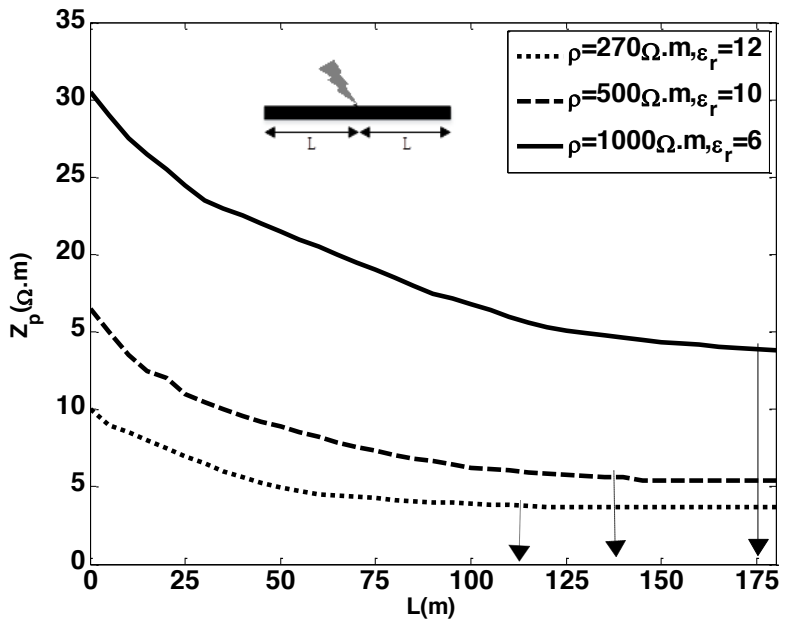

(b)

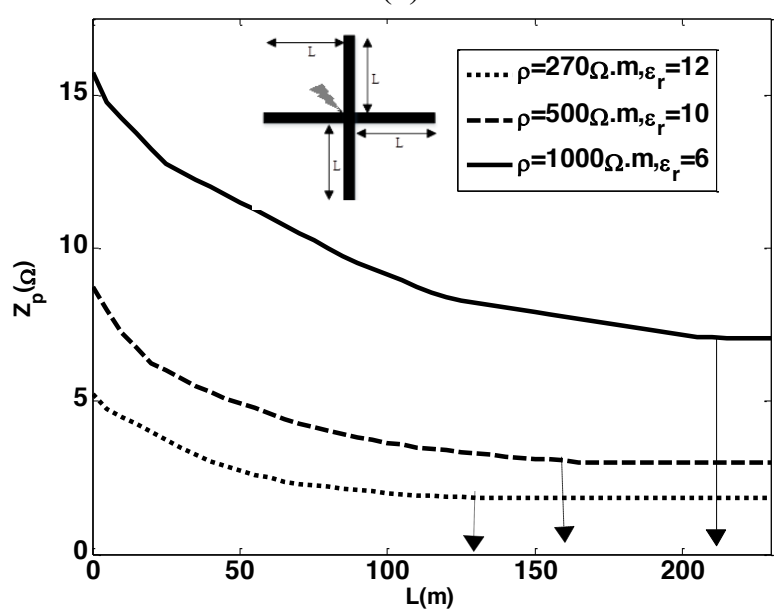

(c)

Figure 8: Impulse impedance of (a): corner-fed electrode, (b): center-fed two-arm electrode, and (c): center-fed four-arm electrode for the three lossy soils.

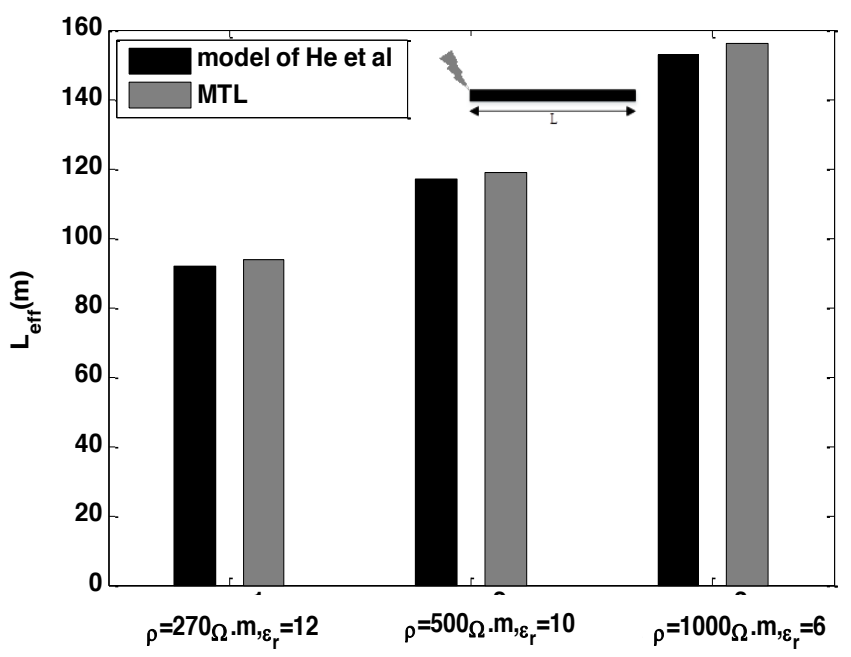

(a) 


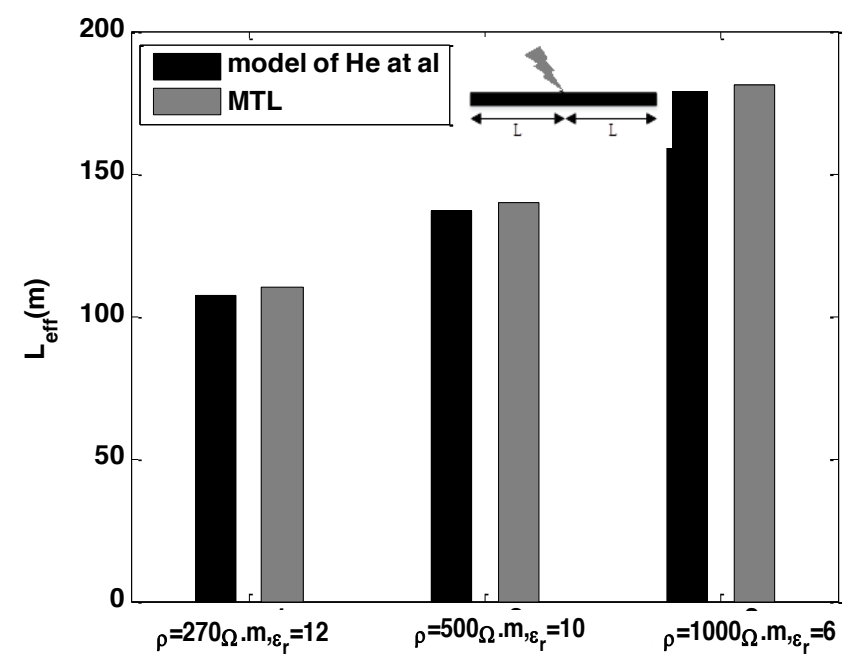

(b)

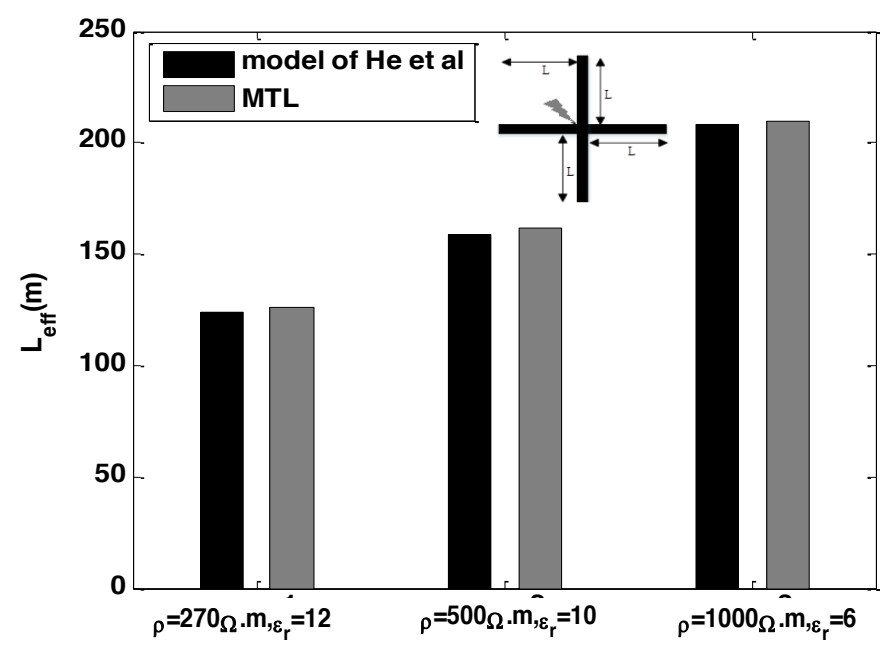

(c)

Figure 9: Comparison of effective lengths for (a): corner-fed electrode, (b): center-fed electrode with two arms, and (c): center-fed electrode with four arms.

As can be observed in figure 9, the effective lengths are in good agreement. Table 1 shows the relative error percentage between the two methods. Comparison shows small relative percentage (less than 5\%) which is acceptable from electromagnetic engineering point of view.

Table 2: Relative error percentage for computing effective length of counterpoise wires by two methods.

\begin{tabular}{c|ccc}
\hline \multirow{2}{*}{$\begin{array}{c}\text { Counterpoise } \\
\text { wires }\end{array}$} & \multicolumn{3}{|c}{ Relative error percentage } \\
\cline { 2 - 4 } & One-arm & Two-arm & Four-arm \\
\hline$\rho=270 \Omega \cdot \mathrm{m}, \varepsilon_{\mathrm{r}}=12$ & $2.1 \%$ & $4.3 \%$ & $3.8 \%$ \\
\hline$\rho=500 \Omega \cdot \mathrm{m}, \varepsilon_{\mathrm{r}}=10$ & $4.2 \%$ & $3.7 \%$ & $3.2 \%$ \\
\hline$\rho=1000 \Omega \cdot \mathrm{m}, \varepsilon_{\mathrm{r}}=6$ & $3.3 \%$ & $2.8 \%$ & $2.4 \%$ \\
\hline
\end{tabular}

\section{Sensitivity Analysis}

In the previous section, the validity of the improved MTL for predicting transient voltage, and effective length of counterpoise wires buried in only-ionized soils was investigated. Evidently, due to its frequency-domain nature, the dispersion effect of soil can be easily incorporated. The dispersion model of soil here used is based on Alipio-Visacro measurement [4], that is

$$
\begin{aligned}
& \rho(f)=\rho_{0}\left(1+\left(1.2 \times 0^{-6} \times \rho_{0}^{0.73}\right) \times(\mathrm{f}-100)^{0.65}\right)^{-1} \\
& \varepsilon_{\mathrm{r}}(\mathrm{f})= \begin{cases}192.2 & \mathrm{f} \leq 10 \mathrm{kHz} \\
1.3+7.6 \times 0^{3} \times \mathrm{f}^{-0.4} & \mathrm{f} \geq 10 \mathrm{kHz}\end{cases}
\end{aligned}
$$

where $\rho_{0}$ is low-frequency resistivity of lossy soil. Frequency variations of resistivity and relative permittivity are shown in figure 10. This figure shows that the soil dispersion is more pronounced for highly resistive soils ( $\rho_{0}>100 \Omega . m$ ) and vice versa.

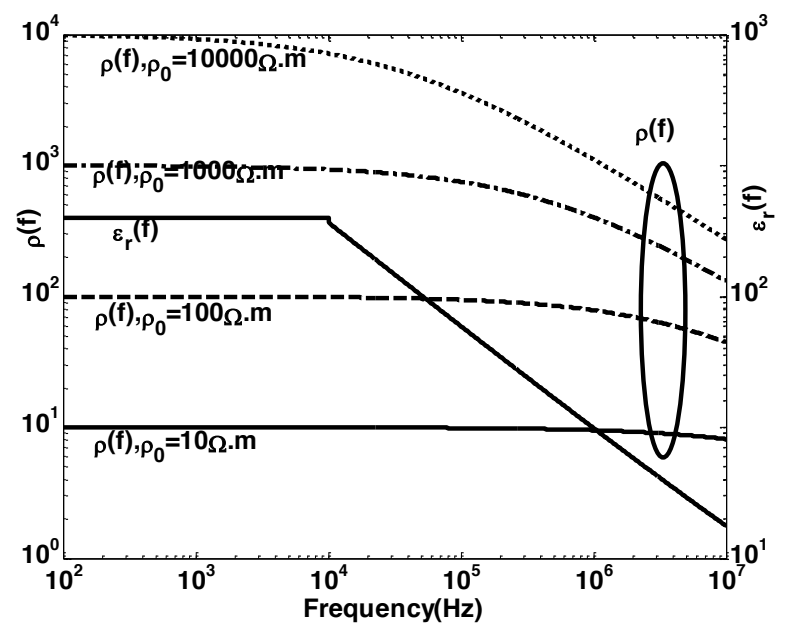

Figure 10: Frequency variation of resistivity (left axis) and relative permittivity (right axis) based on Alipio-Visacro measurement [4].

Ability of the improved MTL for computing transient voltage in both-affected soils was validated [24] (see figure 8 in [24]). However, there is no research on the effective length of counterpoise wires buried in both-affected soils to be validated. Hence, to show the difference between the two situations, i.e. both-affected and only-ionized soils, a sensitivity analysis is carried out using defining the following decrement factor

$\mathrm{DF}=\frac{\mathrm{L}_{\text {eff }} \text { of both }- \text { affected soil }}{\mathrm{L}_{\text {eff }} \text { of only }- \text { ionized soil }}$

Now the effects of three parameters, i.e. low-frequency resistivity of soil $\left(\rho_{0}\right)$, rise time $\left(T_{M}\right)$ and magnitude $\left(I_{M}\right)$ of the lightning current, on the DF are investigated. The simulation results for sensitivity analysis are shown in figures 11,12 and 13 . From these figures, the following key findings are inferred:

1-From figure 11, when the low-frequency-resistivity of soil is increased, the effective length of counterpoise wires buried in both-affected soil become less than the individual one in only-ionized soil especially in highly resistive soils, whereas 
in poorly resistive soils $\left(\rho_{0} \leq 10 \Omega \cdot \mathrm{m}\right)$, since the dispersion effect is decreased, two situations results in approximately the same effective length. In addition, when the number of arms is increased, since the ionization effect is decreased, the decrement factor is less affected.

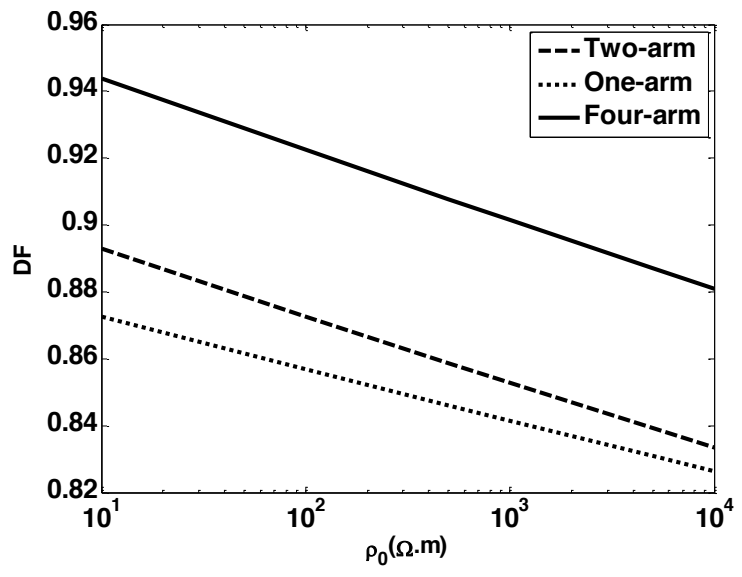

Figure 11: Variation of DF versus $\rho_{0}$ for counterpoise wires.

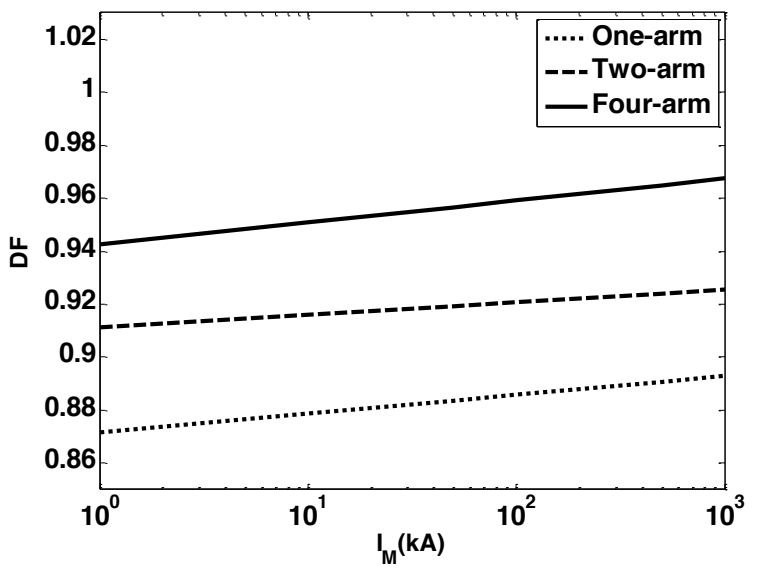

Figure 15: Variation of DF versus $\mathrm{I}_{\mathrm{M}}$ for counterpoise wires. $\left(\rho_{0}=1000 \Omega \cdot \mathrm{m}, \mathrm{T}_{\mathrm{M}}=8 \mu \mathrm{s}\right)$.

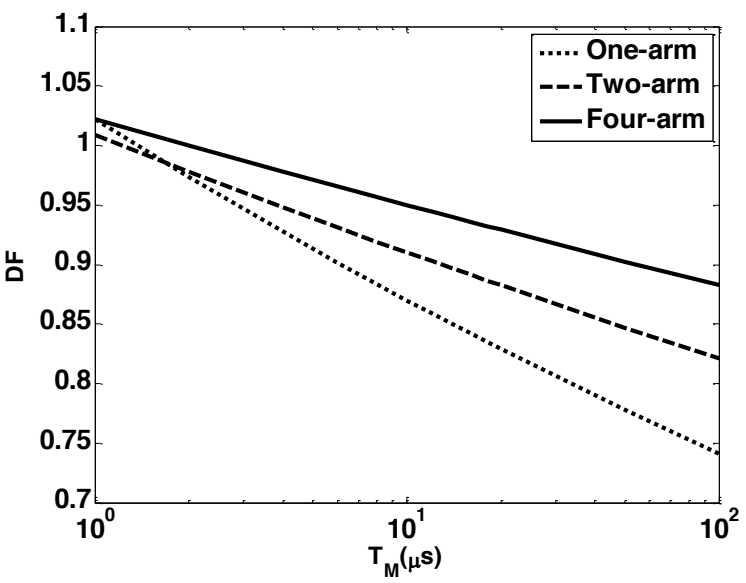

Figure 16: Variation of DF versus $\mathrm{T}_{\mathrm{M}}$ for counterpoise wires. $\left(\rho_{0}=1000 \Omega \cdot \mathrm{m}, \mathrm{I}_{\mathrm{M}}=50 \mathrm{kA}\right)$.
2-With reference to figure 12 , when the magnitude of lightning current is increased, two situations results in approximately the same effective length. This is physically because of decreasing/increasing the soil resistivity/conductivity surrounding the electrodes [1] which results in decreasing dispersion effect. This fact is more pronounced when the number of arms is increased.

3 -As can be seen from figure 13, for low-valued rise times (fast-fronted currents), the two situations results in the same effective length, whereas for high-valued rise times (slowfronted currents) the effective length in both-affected soil is less than the individual one in only-ionized soils. This fact was also observed in only-dispersive soils [18] where the effective length of electrodes under subsequent stroke current is less affected than that of the first stroke current. Physical reason can be found in [2]. The above extracted findings are financially important in power engineering point of view.

Note that the approximate method in [26], i.e. transmission line method in one dimension (TLM-1-D), due to its time-domain nature cannot consider the soil dispersion, whereas it is efficiently incorporated by the proposed modeling approach. In addition, although more recently proposed method in [15] which is based on combining MoM with harmonic balance method (MoM-HBM) considers both effects, it is, however, suffers from time-consuming computations of MoM and Newton's Rophson algorithm in iteration process. These drawbacks are repeated when the weather conditions are changed.

Table 3: Comparing run-times of the different approximate methods for computing transient voltage of the counterpoise wires buried in only-ionized soils.

\begin{tabular}{c|ccc}
\hline \multirow{2}{*}{$\begin{array}{c}\text { Counterpoise } \\
\text { wires }\end{array}$} & \multicolumn{3}{c}{ Run-time (sec) } \\
\cline { 2 - 4 } & One-arm & Two-arm & Four-arm \\
\hline Improved MTL & 1.4 & 1.25 & 1.05 \\
\hline MoM-HBM [15] & $20+163$ & $20+163$ & $20+163$ \\
\hline TLM-1-D [26] & 60 & 45 & 30 \\
\hline
\end{tabular}

Table 4: Comparing run-times of the three different methods for computing transient voltage of the counterpoise wires buried in both-affected soils.

\begin{tabular}{c|ccc}
\hline \multirow{2}{*}{$\begin{array}{c}\text { Counterpoise } \\
\text { wires }\end{array}$} & \multicolumn{3}{c}{ Run-time (sec) } \\
\cline { 2 - 4 } & One-arm & Two-arm & Four-arm \\
\hline Improved MTL & 1.5 & 1.35 & 1.15 \\
\hline MoM-HBM [15] & $22+163$ & $22+163$ & $22+163$ \\
\hline TLM-1-D [26] & $\begin{array}{c}\text { Not } \\
\text { applicable }\end{array}$ & $\begin{array}{c}\text { Not } \\
\text { applicable }\end{array}$ & $\begin{array}{c}\text { Not } \\
\text { applicable }\end{array}$ \\
\hline
\end{tabular}

The run-times of transient voltage for the counterpoise wires buried in only-ionized soils are listed in table 3 . In this table, '20', ' 163 ' and '+' respectively mean the run-times of MoM, HBM and algebraic summation. From this table, one can see that the high computational efficiency of the proposed modeling approach in comparison with MoM-HB. Moreover, the run-times for both-affected soil are listed in table 4 accordingly. All computations were carried out on an Intel (R) Core (TM) i7-4702MQ CPU with 4GB of Ram. 


\section{Conclusion}

In this study using a frequency-domain approach called improved MTL, significance of two aspects of lossy soils namely ionization and dispersion on the effective length of counterpoise wires was investigated. The simulation results show that when both effects are taken place, the effective length is generally decreased especially for highly resistive soils and slow-fronted currents which is is financially of importance. For poorly resistive soils and fast-fronted currents, however, the ionization effect is dominant so that the dispersion effect can be disregarded. The next step is to extract closed-form soluitions for effective length of counterpoise wires buried in both-affected soil using combining the proposed method with optimization algorithms [27-31], or fuzzy inference techniques [32-39] that is in progress.

\section{References}

[1] Jinliang He, "Progress in Lightning Impulse Characteristics of Grounding Electrodes With Soil Ionization," IEEE Transaction on Industry Application, 51: 4924-4933, 2015.

[2] S. Visacro, "What Engineers in Industry Should Know About the response of Grounding Electrodes Subjected to Lightning Currents", IEEE Transaction on Industry Application, 51: 4943-4951, 2015.

[3] M. Akbari, K. Sheshyekani, M. Reza Alemi, "The Effect of Frequency Dependence of Soil Electrical Parameters on the Lightning Performance of Grounding Systems", IEEE Transactions on Electromagnetic Compatibility, . 55: 739-746, 2013.

[4] S. Visacro and Rafael Alipio, "Frequency Dependence of Soil Parameters: Experimental Results, Predicting Formula and Influence on the Lightning Response of Grounding Electrodes", IEEE Transactions on Electromagnetic Compatibility, 27, 927-935, 2012.

[5] R. Alipio, S. Visacro, "Modeling the Frequency Dependence of Electrical Parameters of Soil", IEEE Transactions on Electromagnetic Compatibility, 15, 1163-1171, 2014.

[6] C. M. Portela, M. C. Tavaras, and P. Filho, "Accurate Representation of Soil Behavior for Transient Studies", IEE Proceedings of Generation, Transmission and Distribution, 150: 123-129, 2003.

[7] G. Ala, P. L. Buccheri, P. Romano, and F. Viola, "Finite Difference Time Domain Simulation of Earth Electrodes Soil Ionization under Lightning Surge Conditions," IET Science, Measurement Technology, 2: 134-135, 2008.

[8] Z. Feng, X. Wen, X. Tong, H. Lu, L. Lan, and P. Xing, "Impulse Characteristics of Tower Grounding Devices Considering Soil Ionization by the Time-Domain Difference Method", IEEE Transactions on Power Delivery, 30: 1906-1913, 2015.

[9] J. Cidrás, A. F. Otero, and C. Garrido, "Nodal Frequency Analysis of Grounding Systems Considering the Soil Ionization Effect," IEEE Transactions on Power Delivery, 15, 103-107, 2000.

[10] B. Zhang, J. He, J. Lee, X. Cui, Z. Zhao, and J. Zou, "Numerical Analysis of Transient Performance of Grounding Systems Considering Soil Ionization by Coupling Moment Method with Circuit Theory," IEEE Transactions on Magnetics, 41, 1440-1443, 2005.

[11]B. Zhang, J. Wu, Jinliang He, R. Zeng, "Analysis of Transient Performance of Grounding System Considering Soil Ionization by the Time Domain
Method", IEEE Transactions on Magnetics, 49: $1837-$ 1840, 2013.

[12] J. Wu, B. Zhang, J. He, and R. Zeng, "A Comprehensive Approach for Transient Performance of Grounding System in the Time Domain", IEEE Transactions on Electromagnetic Compatibility, 57: 250-256, 2015.

[13] H. Chen, and Y. Du, "Lightning Grounding Grid Model Considering Both the Frequency-Dependent Behavior and Ionization Phenomenon", IEEE Transactions on Electromagnetic Compatibility, 61: 157-165, 2019.

[14] O. Kherif, S. Chiheb, M. Teguar, A. Mekhaldi,and N. Harid, "Time-Domain Modeling of Grounding Systems' Impulse Response Incorporating Nonlinear and Frequency-Dependent Aspects", IEEE Transactions on Electromagnetic Compatibility, 60: 907-916, 2018.

[15] Jalil Ghayur Safar, Reza Shariatinasab, and Jinliang He, "Comprehensive Modeling of Grounding Electrodes Buried in Ionized Soil Based on MoM-HBM Approach", IEEE Trans. Power. Del. 57: 1627-1636, 2019.

[16] Jinliang He et al, "Effective length of counterpoise wire under lightning current," IEEE Trans. Power Del, 20: 1585-1591, 2005.

[17] Rong Zeng, Xuehai, Jinliang He, Bo Zhang and yanqing gao, "Lightning Impulse performance of Grounding Grids for Substations Considering Soil Ionization", IEEE Transaction on Power Delivery, 23: 324-329, 2008.

[18] R. Alipio, S. Visacro, "Impulse Efficiency of Grounding Electrodes: Effect of Frequency-Dependent Soil Parameters," IEEE Transaction on Power Delivery, 29: 716-723, 2014.

[19]S. Visacro, Rafael Alipio, Clever Pereira, Miguel Guimaraes, and Marcoa. O. Schroeder, "Lightning Response of Grounding Grids: Simulated and Experimental Results", IEEE Transaction on Electromagnetic Compatibility, 57: 121-127, 2015.

[20] L. Grcev, "Impulse efficiency of Ground Electrodes", IEEE Transaction on Power Delivery, 24: 441-451, 2009.

[21]L. Crcev, "Lightning Surge efficiency of Grounding Grids", IEEE Transaction on Power Delivery, 26: 16921699, 2011.

[22] A. Jardines, J. L. Guardado, J. Torres, J. J. Chavez, M. Hernandez, "A Multiconductor Transmission Line Model for Grounding Grid", Electrical Power and Energy Systems, 60: 24-33, 2014.

[23] S. S. Sajjadi, S. R. Ostadzadeh, "Lightning response of Multi-port Grounding Grids Buried in Dispersive Soils: An Approximation versus Full Wave Methods and Experiment", Advanced Electromagnetics, 8: 43-50, 2019.

[24] S. S. Sajjadi, V. Aghajani, S. R. Ostadzadeh, "Transient Analyses of Grounding Electrodes Considering Ionization and Dispersion Aspects of Soils Simultaneously: An Improved Multiconductor Transmission Line Model (Improved MTL)", Applied Computational Electromagnetic Society Journal, 34: 731-737, 2019.

[25] R. S. Bretas, Guilherme A. D. Dias, Marcos Tello, Dave W. P. Thomas, and Christos Christopoulos, "The transmission line modeling method to represent the soil ionization phenomenon in grounding systems", IEEE Transactions on Magnetics, 50: 1163-1171, 2014.

[26] Yaqing Liu, "Investigating the Validity of Existing Definitions and Empirical Equations of Effective Length/Area of Grounding Wire/Grid for Transient Studies," Journal of Electrostatics, 65: 329-335, 2007.

[27] H. Yazdi, S. R. Ostadzadeh and F. Taheri, "Transient Analysis of Single-Conductor Overhead Lines Terminated to Grounded Arrester Considering 
Frequency Dependence of Electrical Parameters of Soil using Genetic Algorithm", Journal of Applied Electromagnetic (JAPEM), 3: 35-42, 2015.

[28] F. Taheri, S. R. Ostadzadeh, "Transient Analysis of the Single-Conductor Overhead Lines Connected to GridGrounded Arrester under Direct Lightning by Means of GA", Journal of Communication Engineering, 3: 45-54, 2014.

[29] M. Teimoori, S. R. Ostadzadeh, "Analysis of Frequency Selective with Nonlinear Antenna under Radiations of Bi-Frequency Waves Based on Genetic Algorithm", International Journal of Computer \& Technology, 15: 6914-6922, 2016.

[30] H. Samiian, S. R. Ostadzadeh, and A. Mirzarie, "Application of Intelligent Water Drops in Transient Analysis of Single Conductor Overhead Lines Terminated to Grid-Grounded Arrester under Direct Lightning Strikes", Journal of Communication Engineering, 5: 50-59, 2016.

[31]A. Bahrami, S. R. Ostadzadeh, "Back Scattering Response from Single, Finite and Infinite Array of Nonlinear Antennas based on Intelligent Water Drops Algorithm”, 38: 2040-2056, 2019.

[32] S. R. Ostadzadeh, S. M. taghavi, M. Kazemi, A. Bagheri, "Closed-Form Solutions for Broad-Band Equivalent Circuit of Vertical Rod Buried in Lossy Grounds Subjected to Lightning Strokes", Journal of Communication Engineering,6: 26-38, 2107.

[33] S. R. Ostadzadeh, M. Tayarani, M. Soleimani, "Prediction of Scattered Field from Linearly Loaded Dipole Antenna using Fuzzy Inference", 2008 International Conference on Computational Intelligence for Modelling Control \& Automation, 941-945, 2008.

[34] S. R. Ostadzadeh, M. Tayarani, M. Soleimani, “A Fuzzy Model for Computing Input Impedance of Two Coupled Dipole Antennas in the Echelon Form", Progress In Electromagnetics Research, 78: 265-283, 2008.

[35] S. R. Ostadzadeh, M. Tayarani, M. Soleimani, “A Fuzzy Fodel for Computing Back-Scattering Response from Linearly, Loaded Dipole Antenna in the Frequency Domain", Progress In Electromagnetics Research, 86: 229-242, 2008.

[36] S. R. Ostadzadeh, M. Tayarani, M. Soleimani, “A Hybrid Model in Analyzing Nonlinearly Loaded Dipole Antenna and Finite Antenna Array in the Frequency Domain", International Journal of $R F$ and Microwave Computer-Aided Engineering, 19: 512-518, 2009.

[37]S. R. Ostadzadeh, "An Efficient Hybrid Model in Analyzing Nonlinearly Loaded Dipole Antenna above Lossy Ground in the Frequency Domain", Applied (ACES), 28: 731-737, 2013.

[38] S. R. Ostadzadeh, "Qualitative Model of the Input Impedance of Rectangular Microstrip Antenna", Journal of Fuzzy Set Valued Analysis, 2:154-165, 2015.

[39] Z. Samiee, S. R. Ostadzadeh, "Transient Analyses of Grounding Systems Subjected by Lightning Surge Currents through Fuzzy-Based Models of Input Impedance in the Frequency Domain", Asian Journal of Fuzzy and Applied Mathematics, 3: 22-34, 2015. 